\title{
Candida Perinephric Abscess: A Rare Presentation in a Trauma Patient
}

\author{
Natesh Yepuri, James Osei Sarpong, and Robert N. Cooney
}

\begin{abstract}
Background: The perinephric space is a closed anatomic space that plays an important role in limiting and transmitting disease processes. Perinephric abscess is an uncommon complication of peritonitis. The development of a perinephric abscess caused by Candida is also rare. In this case report we describe an unusual case of perinephric abscess caused by Candida in a trauma patient. To the best of our knowledge, this is the first case described, and as such, represents a novel case.

Case Presentation: A 17-year-old male was impaled by a tree branch in a go-cart accident and presented to the emergency department with small bowel evisceration through the abdominal wall defect in his left upper quadrant. After he was assessed, he was taken to the operating room for emergency abdominal exploration. Surgical exploration revealed a large gastric laceration with diffuse peritonitis and massive contamination of the peritoneal cavity by gastric contents and minor trauma to the inferior pole of the spleen adjacent to the left kidney. After gastric repair, abdominal lavage, and staged abdominal wall reconstruction, the patient was treated with antibiotics for peritonitis. During the post-operative recovery period the patient became febrile with increasing white blood cell (WBC) count. Abdominal contrast-enhanced computed tomography (CT) revealed fluid accumulation in the left perinephric space, which was drained percutaneously and grew Candida albicans. Conclusion: Candida can cause perinephric abscess in patients with peritonitis and the clinical picture is usually indistinguishable from that caused by bacteria. A better understanding of the patient's risk factors and events as in this case, would presumably result in earlier diagnosis and prompt management.
\end{abstract}

Keywords: computed tomography; perinephric abscess; perinephric space

$\mathbf{T}$ HE PERINEPHRIC SPACE (PS) is a central, well-defined retroperitoneal compartment located between the anterior and posterior pararenal spaces. The PS contains the kidneys, proximal ureters, adrenal glands, bridging septa, vessels, and lymphatics. Acute conditions involving the PS can result from pathologic conditions that occur in isolation, spread from nearby structures, or as a sequelae of a systemic infection [1].

Retroperitoneal fluid collections from infectious, neoplastic, inflammatory, and traumatic conditions are well established. Infections involving the PS are a rare clinical entity and are regarded as dangerous complications of urinary tract infection. A perinephric abscess (PNA) is a collection of suppurative material in the perinephric space between the renal capsule and Gerota fascia, affecting one in 10 cases for every 10,000 hospital admissions, with males and females affected equally [2]. Salvatierra et al. reported a mortality rate of $56 \%$ in a series of 71 cases, whereas Adachi and Carter noted a mortality rate of $39 \%[3,4]$. In both the studies, the high mortality rate is attributed to delay in diagnosis resulting from non-specific clinical findings.

\section{Case Presentation}

The patient was a 17-year old male impaled by a tree branch in a go-cart accident who presented to our trauma center in acute distress because of pain; he was tachycardic but normotensive with stable blood pressure. Abdominal examination revealed diffuse tenderness with rebound and small bowel evisceration in the left upper quadrant through a defect in the lateral abdominal wall. After the initial resuscitation, the patient was scheduled for emergent abdominal exploration. Intra-operative findings are shown in

Department of Surgery, SUNY Upstate Medical University, Syracuse New York.

(C) Natesh Yepuri et al. 2017; Published by Mary Ann Liebert, Inc. This Open Access article is distributed under the terms of the Creative Commons License (http://creativecommons.org/licenses/by/4.0), which permits unrestricted use, distribution, and reproduction in any medium, provided the original work is properly credited. 


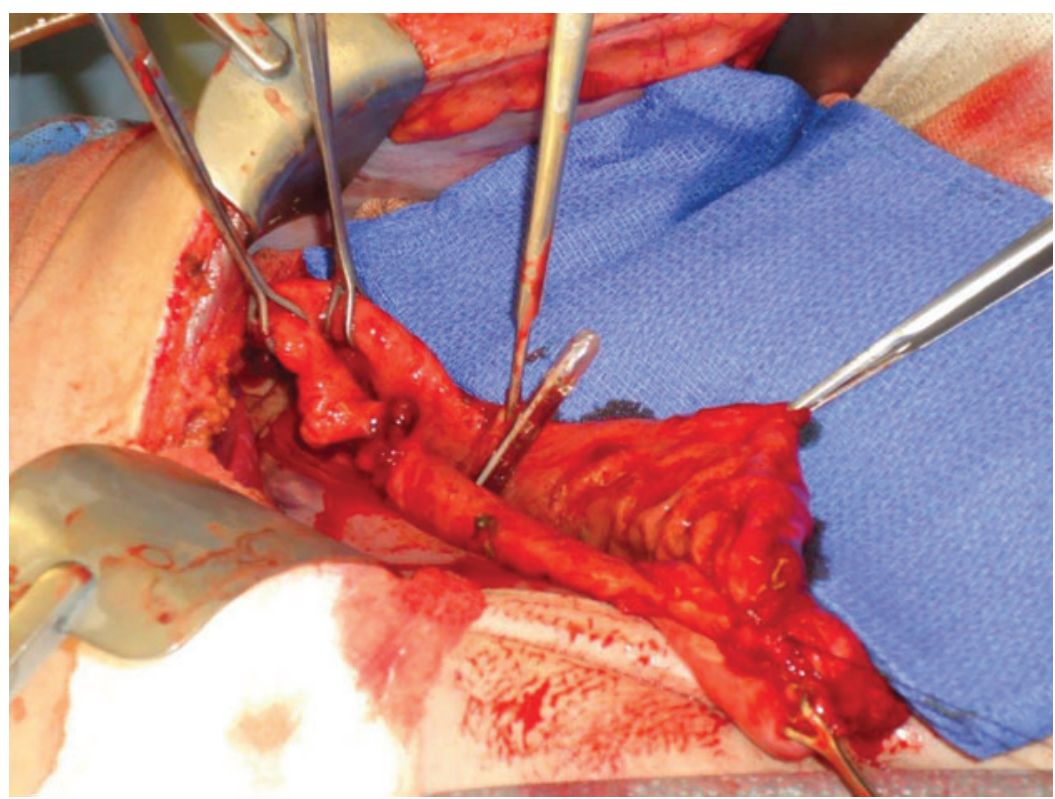

FIG. 1. Nasogastric tube protrusion through stomach laceration.

Figure 1. Surgical exploration revealed hemoperitoneum and diffuse contamination from gastric contents. Bleeding from the inferior pole of the spleen was controlled with packing, and a gastric laceration of 10- to 12-inches in length along the greater curvature extending into the lesser sac was observed. After repair of the gastric laceration, the peritoneal cavity was irrigated with more than five liters of saline. Despite this irrigation, residual food debris was still found, prompting us to leave the abdominal cavity open with a negative pressure dressing and plan for re-exploration the next day.

Abdominal re-exploration the following day revealed persistent turbid fluid and vegetable matter. After further irrigation of the peritoneal cavity, a transgastric jejunostomy tube was established, the abdominal wall defect in the left upper quad-

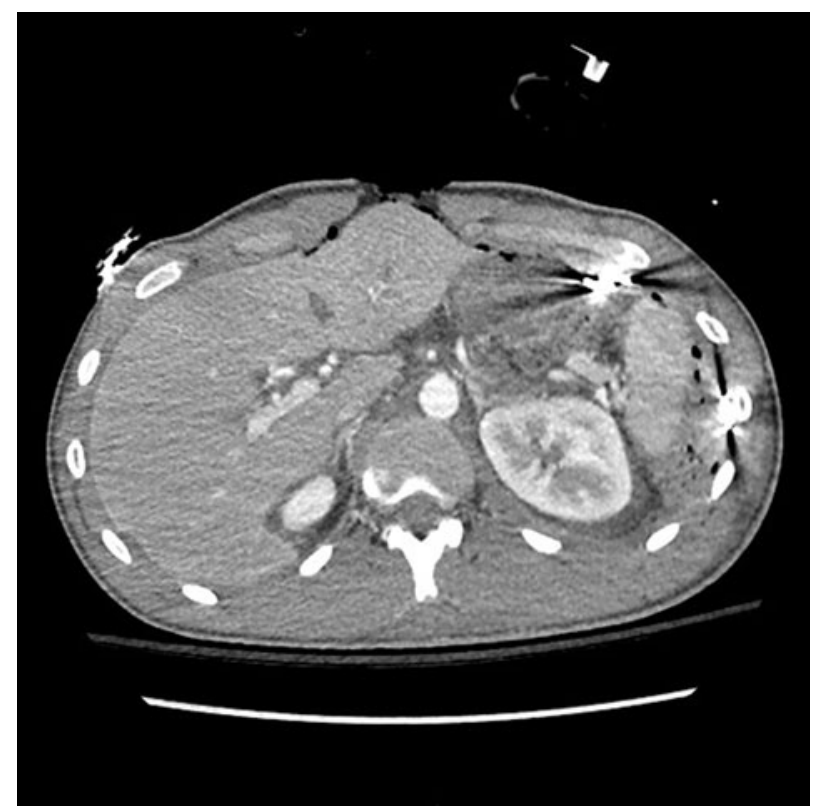

FIG. 2. Left perirenal collection extending into the left psoas. rant was repaired, and the patient was extubated successfully. Broad spectrum antibiotics (piperacillin-tazobactam) were administered before and after surgery.

On the fourth day after surgery, the patient developed lowgrade fevers with rising WBC; workup revealed anemia, hyperglycemia, and metabolic acidosis. The clinical examination was concerning for peritonitis, so a follow-up abdominal CT was performed on post-operative day five. This revealed a left perirenal collection $(9.4 \mathrm{~cm} \times 4.4 \mathrm{~cm})$ encasing the proximal ureters extending into the left psoas without renal parenchyma involvement (Fig. 2). The fluid was drained percutaneously under CT guidance and culture results were positive for Candida. Percutaneous drainage of the PNA and treatment with fluconazole led to resolution of abscess with improvement of clinical, radiologic, and laboratory findings of sepsis.

\section{Discussion}

The normal flora of the gastrointestinal tract is predominantly gram-negative and anaerobic bacteria. Although Candida species are a common commensal of the digestive tract, they become pathogenic during immunodeficiency states and after prolonged antibiotic treatment. The incidence of Candida infections in surgical patients is approximately 5.6 per 1,000 discharges [5], with gastrointestinal surgery being an important risk factor. Studies have shown that Candida species has been isolated from different sites in $22 \%$ of trauma patients treated in critical care who are unresponsive to antibiotics and those in surgical intensive care unit (ICU) for more than three days, with a mortality rate of $30 \%-$ $75 \%$. Candida isolation from the peritoneal cavity is more likely if the source of infection is the upper gastrointestinal (GI) tract rather than the lower GI tract [6]. However, under most circumstances, Candida is cleared quickly. Failure of local and systemic immunity are implicated in the pathogenesis of Candida infections.

The most common mechanism for perinephric abscess is rupture of a renal cortical abscess with subsequent spread to PS rather than direct infection without kidney involvement [2]. 
Common predisposing conditions include: diabetes mellitus, urolithiasis, obstructive uropathy, previous urologic instrumentation or surgery, immunosuppression, and kidney biopsy [7]. However in this case, the patient had none of these established risk factors. Perinephric abscess is caused commonly by pyogenic bacteria, along with enteric gram-negative bacilli with Staphylococcus aureus predominating. Fungi, particularly Candida species, have been reported as rare causes of perinephric abscess, especially in older patients with diabetes mellitus and urinary tract obstruction $[8,9]$.

The presentation of a PNA is often insidious with vague, non-specific signs and symptoms. Common symptoms include fever, chills, dysuria, lethargy, and flank or abdominal pain. Routine laboratory tests are non-specific with leukocytosis, anemia, and pyuria [2]. Blood cultures are often negative with normal urinalysis in one third of cases in which etiology is nonobstructive. Abdominal CT scan is the imaging procedure of choice to characterize perinephric lesions and provides better definition than ultrasonography regarding the extent of disease and its relation to adjacent structures. A PNA is often unsuspected clinically, because the physical examination findings are limited. Successful management of PNA involves percutaneous drainage in conjunction with appropriate antimicrobial therapy. Prompt recognition and treatment are essential because the patients may become anemic or deteriorate clinically; a large burden of retroperitoneal pus is associated with a mortality rate ranging from $21 \%$ to $56 \%$.

Because of the reflection and fusing of Gerota fascia with other fascial entities PNA remains confined. In most circumstances fluids remain in the compartment of origin, unless they accumulate rapidly and exceed capacity resulting in recruitment of fascial planes for decompression $[1,10]$ that determine the direction of fluid spread. Rarely, PNA may be a presentation of infectious focus from the GI tract. Such dissemination from appendicitis, diverticulitis, or perforated colon has been reported [11-13].

On the basis of CT imaging, laboratory findings, and culture results a diagnosis of Candida PNA was made. However, the exact reasons this patient developed a PNA are unclear. We speculate that impalement by the tree branch resulted in diffuse soiling of the peritoneal cavity with gastric contents, blunt trauma to the upper pole of the kidney and spleen with injury to Gerota fascia, immune defects because of host response to severe injury, and contamination of the PS by gastric flora by blood dissemination predisposed this patient to acute presentation with Candida PNA.

\section{Conclusion}

Candida PNA is an uncommon but treatable complication of post-traumatic gastric peritonitis. In a patient with diffuse gastric peritonitis who develops delayed onset post-operative fever and leukocytosis despite adequate antibiotic therapy, intraabdominal abscess and Candida PNA should be considered in the differential diagnosis. Percutaneous drainage and anti-fungal therapy should be considered as the mainstay of treatment.

\section{Author Disclosure Statement}

The authors have no conflicts of interest or disclosures. This work has not been presented previously and is not in consideration for publication by any other journals.

\section{References}

1. Heller MT, Haarer KA, Thomas E, Thaete F. Acute conditions affecting the perinephric space: Imaging anatomy, pathways of disease spread, and differential diagnosis. Emerg Radiol 2012;19:245-254.

2. Gardiner RA, Gwynne RA, Roberts SA. Perinephric abscess. BJU Int 2011;107(Suppl 3):20-23.

3. Salvatierra O Jr, Bucklew WB, and Morrow JW. Perinephric abscess: A report of 71 cases. J Urol 1967;98:296302.

4. Adachi RT, and Carter R. Perinephric abscess: Current concepts in diagnosis and management. Am Surg 1969;35: $72-75$.

5. Meng MV, Mario LA, McAninch JW. Current treatment and outcomes of perinephric abscesses. J Urol 2002;168: 1337-1340.

6. Borzotta AP, Beardsley K. Candida infections in critically ill trauma patients: A retrospective case-control study. Arch Surg 1999;134:657-665.

7. Gupta N. A rare cause of gastric perforation-Candida infection: A case report and areview of the literature. J Clin Diagn Res 2012;6:1564-1565.

8. Hutchison FN, Kaysen GA. Perinephric abscess: The missed diagnosis. Med Clin North Am 1988;72:993-1014.

9. Balkan II, Savas A, Geduk A, et al. Candida glabrata perinephric abscess. Eurasian J Med 2011;43:63-65.

10. High KP, Quagliarello VJ. Yeast perinephric abscess: Report of a case and review. Clin Infect Dis 1992;15:128-133.

11. Gore RM, Balfe DM, Aizenstein RI, Silverman PM. The great escape: Interfascial decompression planes of the retroperitoneum. AJR Am J Roentgenol 2000;175:363-370.

12. Kao CT, Tsai JD, Lee HC, et al., Right perinephric abscess: A rare presentation of ruptured retrocecal appendicitis. Pediatr Nephrol 2002;17:177-180.

13. Kiliç S, Tevfik MR, Ergin H, Baydinç C. Left perinephric abscess caused by Salmonella enteritidis due to colon perforation. J Urol 2003;170:1945.

Address correspondence to:

Dr. Robert N. Cooney

Department of Surgery

Upstate Medical University

750 E. Adams Street, Suite 8141

Syracuse, NY 13210

E-mail: cooneyr@upstate.edu

$\begin{aligned} & \text { Abbreviations Used } \\ & \mathrm{CT}=\text { computed tomography } \\ & \mathrm{GI}=\text { gastrointestinal } \\ & \mathrm{ICU}=\text { intensive care unit } \\ & \mathrm{PNA}=\text { perinephric abscess } \\ & \mathrm{PS}=\text { perinephric space } \\ & \mathrm{WBC}=\text { white blood cell }\end{aligned}$

Cite this article as: Yepuri N, Sarpong JO, Cooney RN (2017) Candida perinephric abscess: A rare presentation in a trauma patient. Surgical Infections Case Reports 2:1, 55-57, DOI: $10.1089 /$ crsi.2017.0012 